\title{
Plastin-3 is a diagnostic and prognostic marker for pancreatic adenocarcinoma and distinguishes from diffuse large B-cell lymphoma
}

\author{
Fei Xiong, Guan-Hua Wu, Bing Wang and Yong-Jun Chen * (D)
}

\begin{abstract}
Background: Altered Plastin-3 (PLS3; an actin-binding protein) expression was associated with human carcinogenesis, including pancreatic ductal adenocarcinoma (PDA). This study first assessed differentially expressed genes (DEGs) and then bioinformatically and experimentally confirmed PLS3 to be able to predict PDA prognosis and distinguish PDA from diffuse large B-cell lymphoma.
\end{abstract}

Methods: This study screened multiple online databases and revealed DEGs among PDA, normal pancreas, diffuse large B-cell lymphoma (DLBCL), and normal lymph node tissues and then focused on PLS3. These DEGs were analyzed for Gene Ontology (GO) terms, Kaplan-Meier curves, and the log-rank test to characterize their association with PDA prognosis. The receiver operating characteristic curve (ROC) was plotted, and Spearman's tests were performed. Differential PLS3 expression in different tissue specimens $(n=30)$ was evaluated by reverse transcription quantitative polymerase chain reaction (RT-qPCR).

Results: There were a great number of DEGs between PDA and lymph node, between PDA and DLBCL, and between PDA and normal pancreatic tissues. Five DEGs (NET1, KCNK1, MAL2, PLS1, and PLS3) were associated with poor overall survival of PDA patients, but only PLS3 was further verified by the R2 and ICGC datasets. The ROC analysis showed a high PLS3 AUC (area under the curve) value for PDA diagnosis, while PLS3 was able to distinguish PDA from DLBCL. The results of Spearman's analysis showed that PLS3 expression was associated with levels of KRT7, SPP1, and SPARC. Differential PLS3 expression in different tissue specimens was further validated by RT-qPCR.

Conclusions: Altered PLS3 expression was useful in diagnosis and prognosis of PDA as well as to distinguish PDA from DLBCL.

Keywords: Pancreatic adenocarcinoma, Bioinformatic analysis, Differentially expressed genes, Biomarker, Diffuse large B-cell lymphoma

*Correspondence: Yjchen@tjh.tjmu.edu.cn

Department of Biliary-Pancreatic Surgery, Tongji Hospital, Tongji Medical College, Huazhong University of Science and Technology, Wuhan 430074, Hubei, China

\section{Introduction}

Pancreatic ductal adenocarcinoma (PDA) is one of the most malignant and lethal cancers, accounting for 459,000 new cases diagnosed in 2018. The overall 5-year PDA survival rate is approximately 9\% [1]. Currently, as the seventh most common cause of cancer death in the USA, PDA is projected to become the second most original author(s) and the source, provide a link to the Creative Commons licence, and indicate if changes were made. The images or other third party material in this article are included in the article's Creative Commons licence, unless indicated otherwise in a credit line to the material. If material is not included in the article's Creative Commons licence and your intended use is not permitted by statutory regulation or exceeds the permitted use, you will need to obtain permission directly from the copyright holder. To view a copy of this licence, visit http://creativecommons.org/licenses/by/4.0/. The Creative Commons Public Domain Dedication waiver (http://creativeco mmons.org/publicdomain/zero/1.0/) applies to the data made available in this article, unless otherwise stated in a credit line to the data. 
common cause of cancer death in the USA by 2030 [2]. The development of PDA is associated with many risk factors that are poorly characterized, rendering PDA prevention almost impossible [1]. Moreover, despite recent advancements in our understanding of the tumor's biology, PDA is still frequently diagnosed at advanced stages of disease (initial diagnosis only occurs in up to $15 \%$ of PDA patients with surgically resectable tumors) [1]. Surgical tumor resection is not possible in many cases, and PDA is insensitive to chemoradiotherapy [3, 4]; however, in early stage PDA, preoperative chemotherapy followed by surgical resection is regarded as a curative treatment [5], but it has not been associated with significant improvement in patient outcomes or quality of life in advanced PDA [6]. Thus, there is an urgent need to understand the molecular mechanisms of PDA carcinogenesis and progression in order to develop biomarkers to diagnose PDA early, to predict prognosis and treatment responses, and to design novel strategies for the control of PDA.

In terms of early PDA detection, the differential diagnosis of an abdominal mass, like PDA, is challenging. Primary pancreatic lymphoma (PPL) is an extremely rare form of extranodal malignant lymphoma, accounting for less than $0.5 \%$ of pancreatic neoplasms and $1 \%$ of extranodal lymphomas [7]. The most common histological type of PPL is diffuse large B cell lymphoma (DLBCL), which accounts for nearly $60 \%$ of all PPL cases [8]. PPL manifests as an abdominal mass that is similar to PDA [9]; however, chemotherapy (like the CHOP regimen) is the preferred treatment option for PPL patients, so the differential diagnosis is crucial. However, if PPL was misdiagnosed, the patients would undergo unnecessary surgery, which is not an ideal treatment option for the patients [10-12].

Plastin-3 (PLS3), belonging to a family of actin-binding proteins, functions to inhibit cofilin-mediated depolymerization of the actin fiber, which may have an important role in the epithelial-mesenchymal transition (EMT) [13]. Altered PLS3 expression was reported to be associated with human carcinogenesis, including PDA, and the detection of PLS3 expression may predict prognosis in various human cancers [13-19]. Molecularly, PLS3 regulates the PI3K/AKT signaling pathway and the EMT in tumor cells $[13,14]$.

In this study, we performed bioinformatical analyses of multiple datasets to assess differentially expressed genes (DEGs) in PDA, normal pancreas, DLBCL, and normal lymph node tissue. We then focused on PLS3 as a biomarker for the early diagnosis of PDA, as well as the prediction of prognosis and differential diagnosis from DLBCL. We expect to provide useful information regarding PLS3 as a biomarker for PDA, in future validation studies.

\section{Materials and methods \\ Searching and downloading of microarray data}

In this study, we searched and downloaded multiple data from various databases [i.e., the Gene Expression Omnibus (GEO; https://www.ncbi.nlm.nih.gov/geo), the Metabolic gEne RApid Visualizer (MERAV, http://merav.wi. mit.edu), The Cancer Genome Atlas (TCGA; https://portal.gdc.cancer.gov/), and the UCSC Xena (https://xena. ucsc.edu/)] using our search criteria (Fig. 1). The GEO datasets included GSE16515, GSE15471, GSE32676, GSE71989, GSE71729, GSE62165, and GSE62452, while GSE16515, GSE2109, and GSE7307 were used for batchnormalization with the Metabolic Gene Rapid Visualizer (MERAV; http://merav.wi.mit.edu/). The microarray data on PDA and DLBCL cell lines were downloaded from the MERAV [20], and microarray data from TCGA and the Genotype-Tissue Expression (GTEx) were obtained from the UCSC Xena database (https://xenabrowser.net/). Each dataset contained various numbers of PDA, normal pancreas, lymph node, and DLBCL tissue samples (Tables 1 and 2).

\section{Identifications of DEGs}

To identify the DEGs, we processed the downloaded database data with the "limma" $\mathrm{R}$ package using the $\mathrm{R}$ version 3.6.3 (https://www.r-project.org) according to a previous study [21]. The false-positive results were corrected by adjusting the P-values (adj. P) during Benjamini-Hochberg analysis. The fold-change value was obtained from the logarithm ( $\operatorname{logFC}$ ) analysis. The threshold for each DEG was set as an adj. $\mathrm{P}<0.05$ and $|\operatorname{logFC}|>1$. The R package "sva" was used to adjust batch effects among the GSE16515, GSE15471, GSE32676, and GSE71989 datasets [22]. DEGs were visualized with the "pheatmap" R package. An online Venn diagram (http:// bioinformatics.psb.ugent.be/webtools/Venn/) tool was used to identify intersections among gene sets.

\section{Gene ontology (GO) analysis}

GO analysis was applied to define genes and their products (mRNA or proteins), to identify unique biological properties of high-throughput transcriptome or genome data. These analyses were conducted with the "clusterProfiler" R package. With the cut-off criterion for a significant function was set as an adj. $\mathrm{P}<0.05$ [23], the GO terms were classified into three groups: biological processes (BP), cellular components (CC), and molecular functions (MF). Data were plotted with the "GOplot" $\mathrm{R}$ package [24]. 
Downloading expression microarrays from MERAV

Identification of the DEGs and the expression of these DEGs in pancreas is significantly higher than in lymph nodes and DLBCL
Downloading expression microarrays of GSE16515, GSE15471, GSE32676 and GSE71989

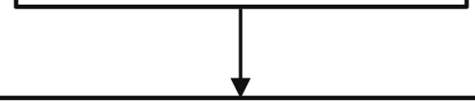

Identification of the DEGs and the expression of these DEGs in

PDA is significantly higher than in pancreas

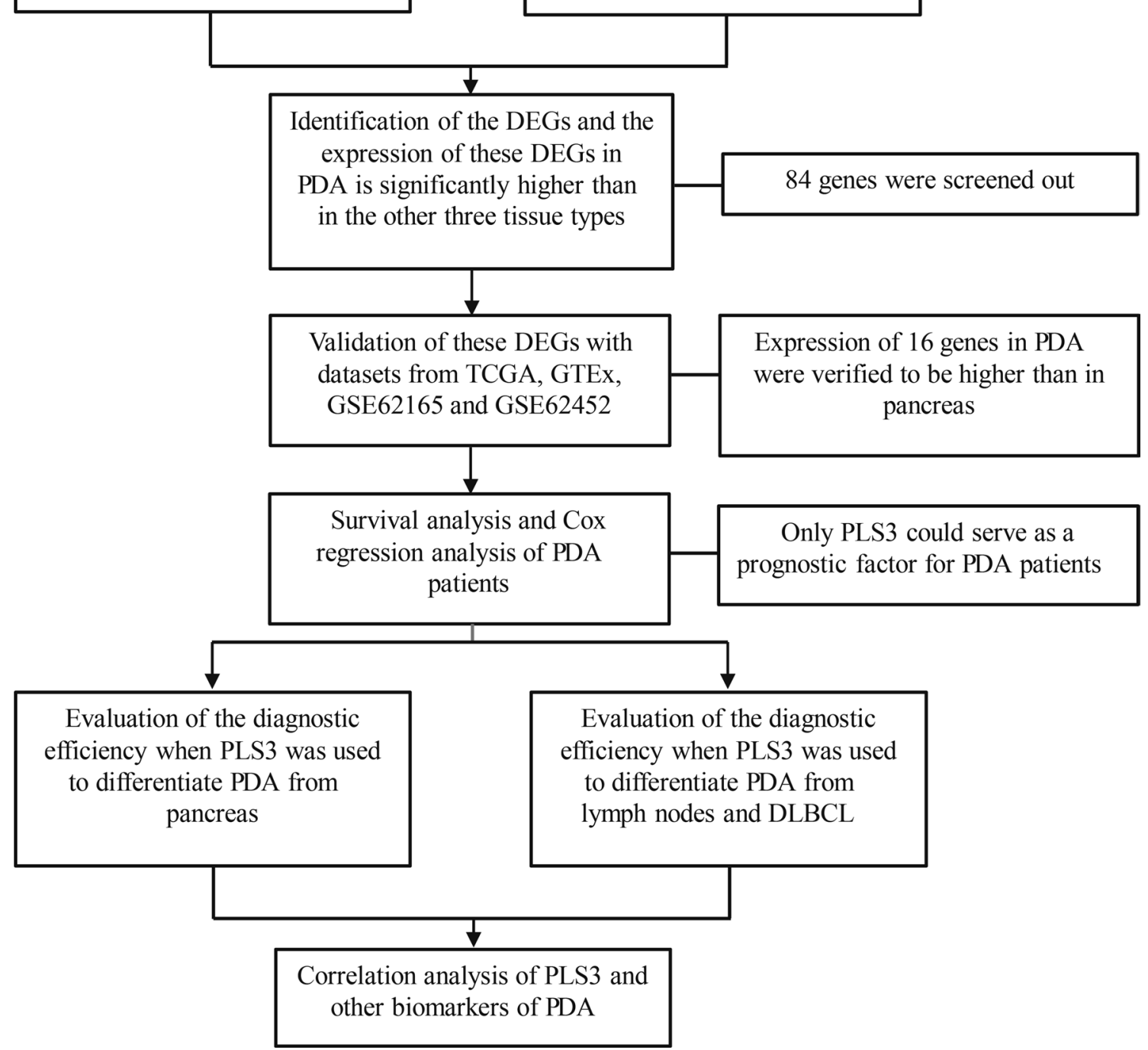

Fig. 1 Illustration of our search criteria and flow diagram

Tissue samples, RNA isolation, and reverse transcription quantitative polymerase chain reaction (RT-qPCR)

Ten pairs of PDA and normal pancreas samples and five pairs of DLBCL and normal lymph node samples were obtained from Tongji Hospital of Huazhong University of Science and Technology, Wuhan, China. This study 
Table 1 The GEO datasets used in this study

\begin{tabular}{lllcl}
\hline Accession number & Platform & Pancreas & PAAD & Lymph nodes \\
\hline GSE15471 & GPL570 & 39 & 39 & 0 \\
GSE16515 & GPL570 & 16 & 36 & 0 \\
GSE32676 & GPL570 & 7 & 25 & 0 \\
GSE71989 & GPL570 & 8 & 13 & 0 \\
GSE71729 & GPL20769 & 46 & 145 & 10 \\
GSE62165 & GPL13667 & 13 & 118 & 0 \\
GSE62452 & GPL6244 & 61 & 69 & 0
\end{tabular}

was approved by the Ethics Committee of Tongji Hospital (detailed in Additional file 1: Table S1). Tissue samples were subjected to total RNA isolation using the RNA isolater Total RNA Extraction Reagent (Vazyme, Nanjing, China) and reverse-transcribed into cDNA using the HiScript III RT SuperMix for qPCR (+gDNA wiper) (Vazyme) according to the manufacturer's instructions. qPCR was then performed using the ChamQ Universal SYBR qPCR Master Mix (Vazyme) in the $\mathrm{iQ}^{\mathrm{TM}}{ }^{\mathrm{T}}$ quantitative PCR detection system (Bio-Rad, Richmond, CA, USA). The primers were: PLS3, 5'-AAGACCTTCCGC AAAGCAATC- $3^{\prime}$ and $5^{\prime}$-TGTTCCTTCGCTGGACAA CTC- $3^{\prime}$, and ACTB, 5'-GTCCACCGCAAATGCTTC TA- $3^{\prime}$ and $5^{\prime}$-TGCTGTCACCTTCACCGTTC- $3^{\prime}$. The qPCR data were quantified using the $2^{-\Delta \Delta \mathrm{Ct}}$ method.

\section{Statistical analyses}

To assess bivariate correlations between variables, we determined Spearman's rank correlation coefficient $\left(\mathrm{r}_{\mathrm{s}}\right)$ using the R 3.6.3 package and SPSS 21.0 (SPSS Inc., Chicago, IL, USA). The output results were visualized using the "corrplot" $\mathrm{R}$ package, and $\mathrm{P}<0.05$ was considered as statistically significant. Kaplan-Meier curves were plotted for 176 PDA patients, the data of which were obtained from TCGA and downloaded from the UCSC Xena site (https://xena.ucsc.edu), then analyzed with the log-rank test to calculate overall survival for groups of patients after stratification for DEGs. Another dataset was downloaded from the complementary data available on the R2: Genomics Analysis and Visualization Platform (http://r2.amc.nl) and International Cancer Genome Consortium (ICGC; https://dcc.icgc.org/) [25]. The data were analyzed by using for Kaplan-Meier analysis with the "survival" $\mathrm{R}$ package. For data analyses, all patients were divided into high vs. low groups, depending on the median expression level of each DEG (cut-off $\mathrm{P}<0.05$ ), using the log-rank test. We also performed univariate and multivariate Cox regression analyses using the "survival" R package. The association between PLS3 expression and clinicopathological features was analyzed with a Chi-square test $(\mathrm{P}<0.05)$. To predict the utility of PLS3 in diagnosing PDA, we plotted the receiver operating characteristic curve (ROC) with the "pROC" R package [26], then calculated the area under the curve (AUC) with SPSS 21.0.

\section{Results}

\section{Identification of PDA-related DEGs using various online datasets}

In this study, we downloaded multiple datasets and performed bioinformatic analyses to identify DEGs in PDA, normal pancreas, DLBCL, and normal lymph node tissue. With use of the MERAV dataset, we found a total of 1611 DEGs between pancreatic and lymph node samples and 3063 DEGs between pancreatic and DLBCL samples (Fig. 2A, B and Table 3). Using 113 PDA and 70 pancreas samples, we compared the DEGs identified with the MERAV dataset with those identified with the GEO dataset (GSE16515, GSE15471, GSE32676, and GSE71989) (Table 1). This approach ultimately resulted in the identification of 1881 upregulated DEGs and 128 downregulated DEGs (Table 3 and Fig. 3A).

We created a search filter for these DEGs in PDA from normal pancreas, lymph node, and DLBCL using the intersection calculation and the transitivity of inequality relation of gene expression sourcing from GEO and MERAV for the "Pancreas $>$ Lymph Nodes", "Pancreas $>$ DLBCL", and "PDA $>$ Pancreas" groups. The intersection showed that 84 DEGs were significantly higher in PDA than in the other three tissue types (Fig. 3B); however, we did not identify any DEGs among the other three groups (Fig. 3C).

Table 2 The MERAV data

\begin{tabular}{lllll}
\hline Tissue & Source & Platform & Number & Sample ID \\
\hline Pancreas & GSE16515 & GPL570 & 15 & $\begin{array}{c}\text { GSM414928, GSM414930, GSM414934, GSM414938, GSM414940, } \\
\text { GSM414942, GSM414947, GSM414953, GSM414955, GSM414957, }\end{array}$ \\
& & & GSM414963, GSM414966, GSM414970, GSM414972, GSM414975 \\
Gymph nodes & GSE7307 & GPL570 & 1 & GSM175950 \\
DLBCL & GSE2109 & GPL570 & 4 & GSM176431, GSM176432, GSM176432, GSM176434 \\
\hline
\end{tabular}



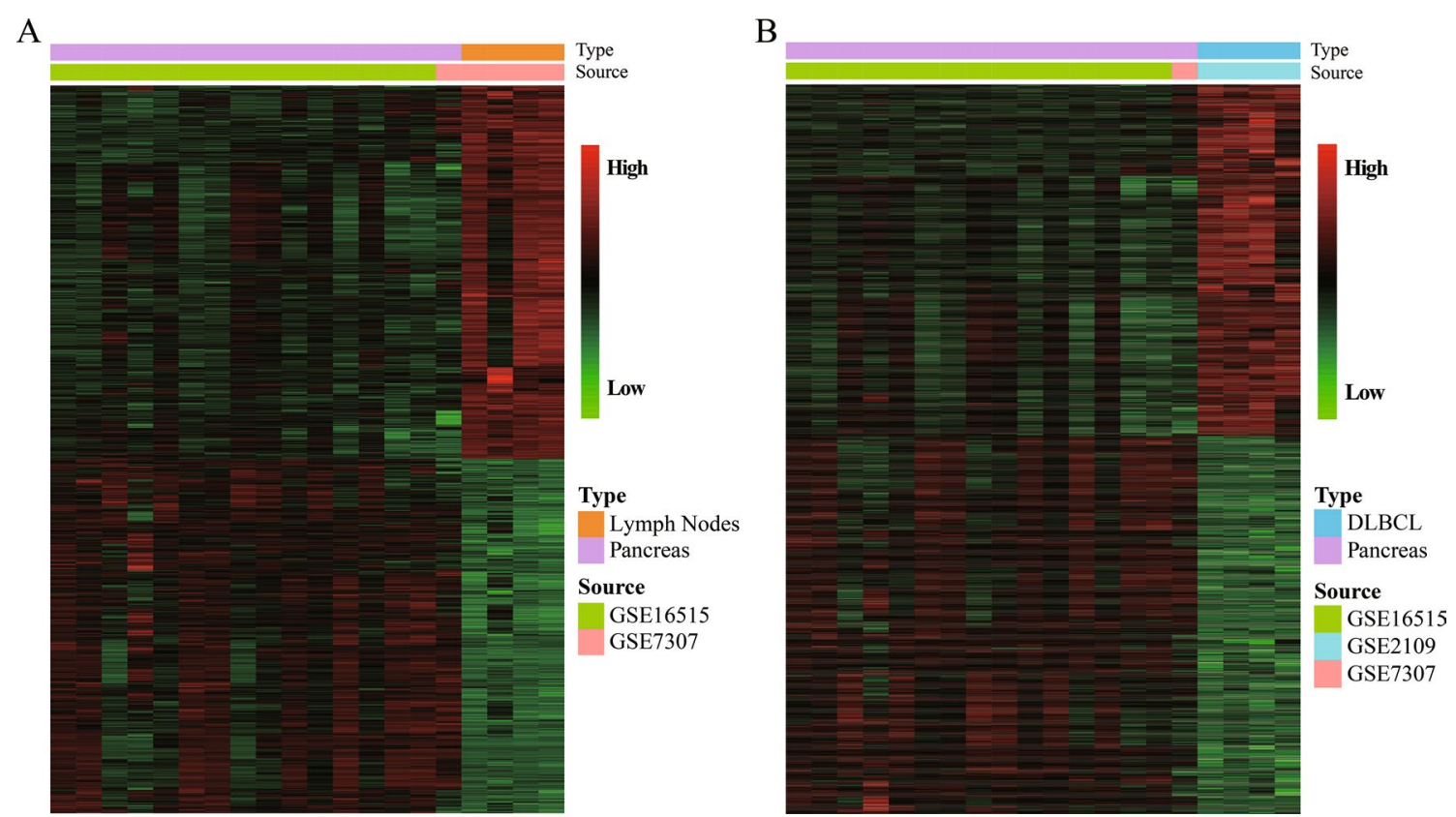

Fig. 2 The hierarchical cluster heatmaps for DEGs in the MERAV datasets. A DEGs between normal pancreas and lymph node tissue. B DEGs between normal pancreas and DLBCL tissue. The gradual change from red to green represents changes in gene expression from high to low. The black color refers to no difference in gene expression. DEG differentially expressed gene, MERAV the Metabolic Gene Rapid Visualizer, DLBCL diffuse large B-cell lymphoma

Table 3 DEGs among PDA, normal pancreas, lymph node, and DLBCL tissues

\begin{tabular}{llcl}
\hline & $\begin{array}{l}\text { Differences in expression between } \\
\text { samples }\end{array}$ & Number of DEGs & Total number \\
\hline Pancreas vs. lymph nodes & Pancreas $>$ lymph nodes & 785 & 1611 \\
& Pancreas $<$ lymph nodes & 826 & 1476 \\
Pancreas vs. DLBCL & Pancreas $>$ DLBCL & 1587 & 3063 \\
& Pancreas $<$ DLBCL & 1881 & 2009 \\
PDA vs. pancreas & PDA $>$ pancreas & 128 & 2 \\
\hline
\end{tabular}

$D E G$ differentially expressed gene, $D L B C L$ diffuse large B-cell lymphoma, $P D A$ pancreatic ductal adenocarcinoma

\section{Functional GO term analysis of these DEGs}

We focused solely on these 84 DEGs for the GO term analysis and found that the top six terms ("cell-cell junction", "cell adhesion molecule binding", "apical part of cell", "lateral plasma membrane", and "desmosome") were significantly associated with PDA development (Fig. 4 and Table 4).

\section{Validation of these DEGs using TCGA and GTEx data}

To validate the DEGs from the MERAV and GEO datasets, we searched and downloaded mRNA sequencing data on 178 PDA and 171 normal pancreas samples from TCGA and the GTEx database. Among a total of 2971 upregulated DEGs in the MERAV and GEO datasets, 46 were confirmed as highly expressed in PDA in TCGA and GTEx datasets. These DEGs may serve as indicators for differentiation between PDA and the other three tissue types (Fig. 5A). An intersectional analysis of GSE62165 (13 pancreas and 118 PDA samples) and GSE62452 (61 pancreas and 69 PDA samples) revealed that 16 of these 46 DEGs were significantly overexpressed in PDA (Fig. 5B and Table 1).

\section{Association of DEGs with PDA prognosis}

We investigated the association of these 16 DEGs with PDA prognosis by plotting the Kaplan-Meier curves and performing the log-rank test on data for the 176 
A
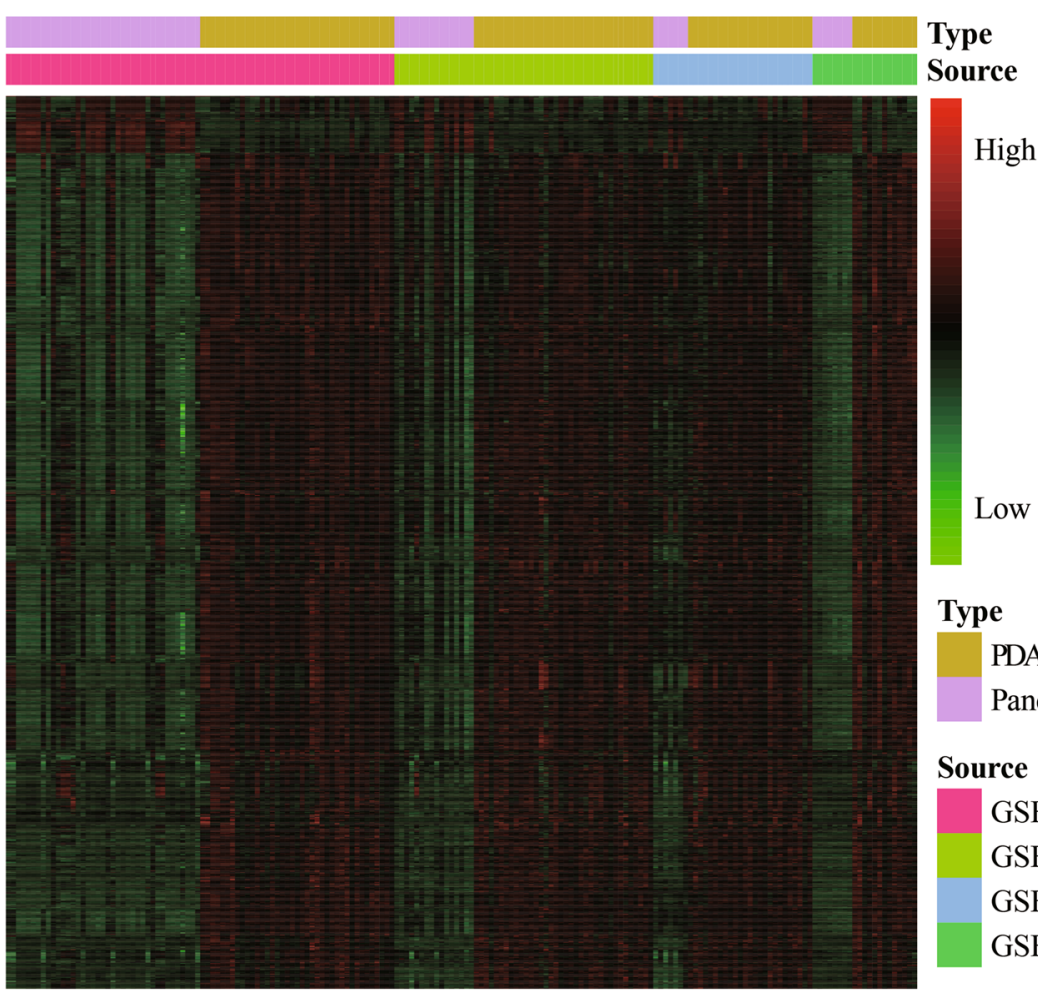

Low

Type

PDA

Pancreas

\section{Source}

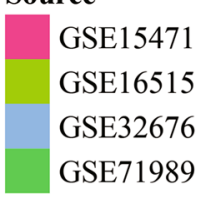

B

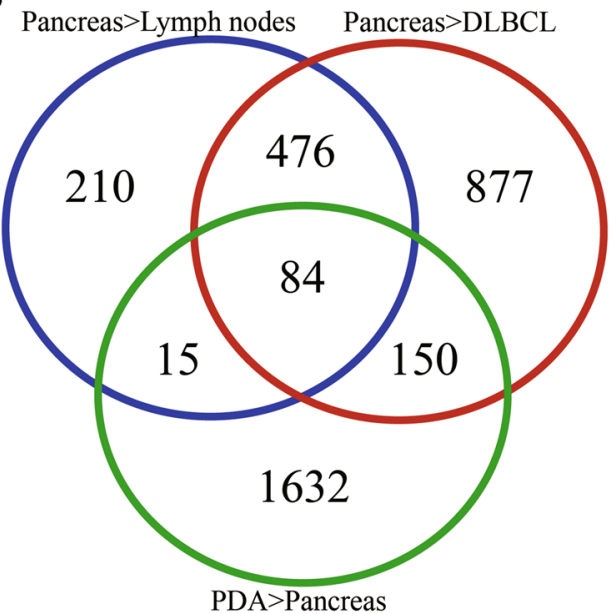

$\mathrm{C}$

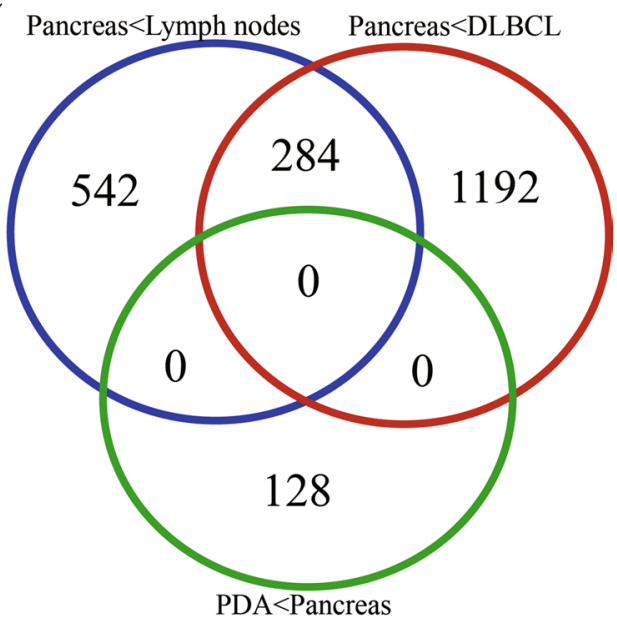

Fig. 3 DEGs identified from the batched GEO datasets with the Venn diagrams. A The hierarchical cluster heatmaps of DEGs between PDA and normal pancreas from the four GEO batched datasets. The gradual change from red to green represents changes in gene expression from high to low. The black color indicates no difference in gene expression. B The intersection among "Pancreas $>$ Lymph Nodes", "Pancreas $>$ DLBCL" and "PDA > Pancreas" groups. C The intersection among "Pancreas < Lymph Nodes", "Pancreas < DLBCL" and "PDA < Pancreas" groups. DEG differentially expressed gene, GEO Gene Expression Omnibus, PDA pancreatic ductal adenocarcinoma, DLBCL diffuse large B-cell lymphoma

PDA patients obtained from TCGA (Additional file 1: Table S1). Our data showed that five (NET1, KCNK1, MAL2, PLS1, and PLS3) of these 16 DEGs were associated with poor overall survival (OS) in PDA patients (Fig. 6A, C, E, G, and I). However, only PLS3 data were verified by the survival data integrated from the R2 and ICGC databases (Additional file 1: Table S1). We also had contradictory data on PLS1, i.e., data from TCGA showed that PLS1 expression was associated with poor survival, but data from another dataset showed the opposite result, 


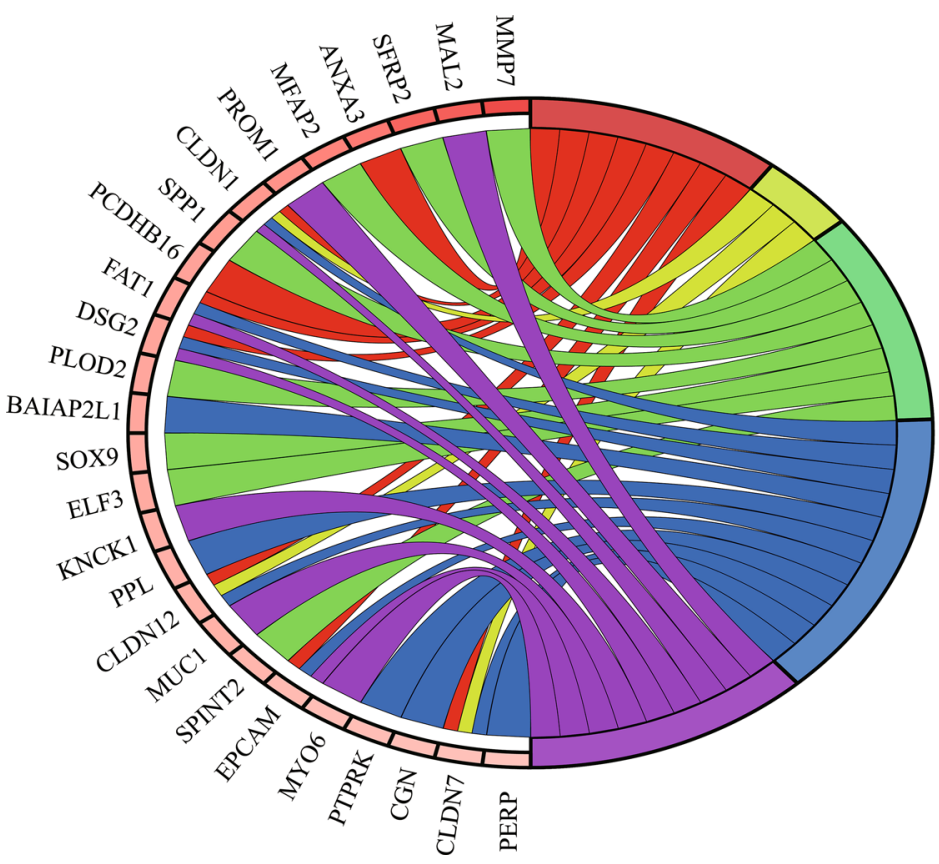

\author{
GO Terms \\ Cell-cell adhesion via plasma-membrane \\ adhesion molecules \\ Calcium-independent cell-cell adhesion via \\ plasma membrane cell-adhesion molecules \\ Extracellular matrix organization \\ Apical part of cell \\ Cell-cell junction
}

$\log \mathrm{FC}$

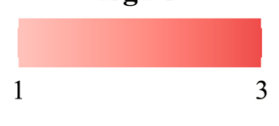

Fig. 4 Gene Ontology terms of the 84 DEGs identified with interaction analysis. Changes in red color saturation represent differences in logFC values

that a decrease in PLS1 expression was associated with poor patient survival (Fig. 6B, D, F, H, J). In this regard, we removed the PLS1 data from our subsequent data analyses. The tumor $\mathrm{N}$ classification was reversely associated with survival of PDA patients (Additional file 2: Figure S1), but we didn't find a correlation between PLS3 expression and the clinicopathological features of PDA (Table 5). The results of univariate and multivariate Cox analyses showed that PDA N classification $(\mathrm{P}=0.004)$ and PLS3 expression $(\mathrm{P}=0.037)$ were significant risk factors in developing PDA (Additional file 1: Table S1). The results of multivariate analysis showed that PDA $\mathrm{N}$ classification $(P=0.036)$ and PLS3 expression $(P=0.026)$ were independent predictors of PDA survival (Table 6).

\section{Accuracy of PLS3 expression in the diagnosis of PDA}

To assess the diagnostic value of PLS3 expression for PDA, we plotted ROC curves and found that PLS3 expression was significantly elevated in PDA (Fig. 7A-D). The diagnostic efficiency of PLS3 in distinguishing PDA from normal pancreas was moderate, with AUC 0.7-0.9 in GSE62452 and high (0.9-1.0) in the other three datasets (Fig. 7E-H).

We found that PLS3 expression was higher in pancreas than in lymph nodes in the MERAV data (Fig. 8A). The diagnostic efficiency of PLS3 was moderate (Fig. 8B). The examination of 46 normal pancreas and 10 normal lymph node samples revealed a similar result for GSE71729 (Fig. 8C, D and Table 1).
The diagnostic value of PLS3 expression was used to differentiate pancreas from DLBCL in 171 pancreas samples from the TCGA and GTEx, 16 pancreas samples from the MERAV, 48 DLBCL samples from the TCGA, and 4 DLBCL samples from the MERAV. Our data showed that PLS3 was overexpressed in normal pancreas and that the diagnostic efficiency of PLS3 was high (Fig. 8E-H). To verify higher PLS3 expression in PDA than in DLBCL and lymph nodes, we analyzed expression data from GSE71729 (145 PDA and 10 lymph node samples) and cell line data from the MERAV (58 PDA and 17 DLBCL samples). The results revealed that PLS3 expression in PDA was dramatically higher than in DLBCL and normal pancreatic tissues (Fig. 5B, 8I, K) and that the diagnostic efficiency of PLS3 was high (Fig. 8J, L). These data indicate that PLS3 could serve as an effective diagnostic marker to differentiate PDA not only from normal pancreas but also from DLBCL and lymph nodes.

\section{Association of PLS3 with known prognostic and diagnostic markers in PDA}

To further elucidate the role of PLS3 expression in PDA, we selected various biomarkers that were previously used to diagnosis PDA or to predict prognosis in affected patients [27-29] and calculated the $r_{s}$ values. Using the batched dataset from GEO data, we found that KRT7 (also known as CK7) and SPP1 (secreted phosphoprotein 1) were associated with PLS3 expression (Fig. 9A), 
Table 4 The remarkably enriched GO terms for these DEGs

\begin{tabular}{|c|c|c|c|c|c|}
\hline Ontology & ID & Description & Count $^{a}$ & adj. $P$ & Gene ID \\
\hline CC & GO:0005911 & Cell-cell junction & 12 & $6.95 E-05$ & $\begin{array}{l}\text { PERP/PPL/EPCAM/FAT1/CLDN1/BAIAP2L1/DSG2/ } \\
\text { CLDN7/CLDN12/PTPRK/CGN/PDLIM3 }\end{array}$ \\
\hline MF & GO:0050839 & Cell adhesion molecule binding & 11 & 0.002369034 & $\begin{array}{l}\text { SFRP2/PPL/SPP1/EPCAM/OLFM4/BAIAP2L1/ } \\
\text { TSPAN8/DSG2/CCN2/PROM1/CGN }\end{array}$ \\
\hline CC & GO:0045177 & Apical part of cell & 9 & 0.002472218 & $\begin{array}{l}\text { EPCAM/FAT1/CLDN1/MYO6/MUC1/DSG2/KCNK1/ } \\
\text { PROM1/MAL2 }\end{array}$ \\
\hline CC & GO:0016324 & Apical plasma membrane & 8 & 0.002817074 & $\begin{array}{l}\text { EPCAM/FAT1/CLDN1/MUC1/DSG2/KCNK1/ } \\
\text { PROM1/MAL2 }\end{array}$ \\
\hline CC & GO:0016328 & Lateral plasma membrane & 4 & 0.003551437 & EPCAM/CLDN1/DSG2/CLDN12 \\
\hline CC & GO:0030057 & Desmosome & 3 & 0.004170446 & PERP/PPL/DSG2 \\
\hline CC & GO:0005923 & Bicellular tight junction & 5 & 0.004170446 & EPCAM/CLDN1/CLDN7/CLDN12/CGN \\
\hline CC & GO:0070160 & Tight junction & 5 & 0.004305753 & EPCAM/CLDN1/CLDN7/CLDN12/CGN \\
\hline BP & GO:0030198 & Extracellular matrix organization & 10 & 0.006175832 & $\begin{array}{l}\text { MMP7/SFRP2/SOX9/SPP1/PLOD2/MFAP2/ELF3/ } \\
\text { CCN2/SPINT2/CCDC80 }\end{array}$ \\
\hline CC & GO:0043296 & Apical junction complex & 5 & 0.006294613 & EPCAM/CLDN1/CLDN7/CLDN12/CGN \\
\hline BP & GO:0043062 & Extracellular structure organization & 10 & 0.010219773 & $\begin{array}{l}\text { MMP7/SFRP2/SOX9/SPP1/PLOD2/MFAP2/ELF3/ } \\
\text { CCN2/SPINT2/CCDC80 }\end{array}$ \\
\hline BP & GO:0098742 & $\begin{array}{l}\text { Cell-cell adhesion via plasma-membrane adhe- } \\
\text { sion molecules }\end{array}$ & 8 & 0.011695026 & $\begin{array}{l}\text { ANXA3/EPCAM/FAT1/CLDN1/DSG2/CLDN7/ } \\
\text { CLDN12/PCDHB16 }\end{array}$ \\
\hline CC & GO:0005884 & Actin filament & 4 & 0.020148378 & PLS3/MYO6/PLS1/PDLIM3 \\
\hline MF & GO:0001968 & Fibronectin binding & 3 & 0.023328786 & $\mathrm{SFRP} 2 / \mathrm{CCN} 2 / \mathrm{CCDC} 80$ \\
\hline CC & GO:0062023 & Collagen-containing extracellular matrix & 7 & 0.023568462 & SFRP2/LGALS4/ASPN/MFAP2/CCN2/F3/CCDC80 \\
\hline MF & GO:0003779 & Actin binding & 8 & 0.042702752 & $\begin{array}{l}\text { PLS3/BAIAP2L1/MYO6/PLS1/ENC1/CGN/MLPH/ } \\
\text { PDLIM3 }\end{array}$ \\
\hline MF & GO:0002162 & Dystroglycan binding & 2 & 0.044071511 & AGR2/AGR3 \\
\hline $\mathrm{BP}$ & GO:0016338 & $\begin{array}{l}\text { Calcium-independent cell-cell adhesion via } \\
\text { plasma membrane cell-adhesion molecules }\end{array}$ & 3 & 0.047603714 & CLDN1/CLDN7/CLDN12 \\
\hline
\end{tabular}

$B P$ biological processes, $C C$ cellular component, $M F$ molecular functions

a The "Count" refers to the numbers of DEGs that were enriched in the corresponding functional category

\section{A}

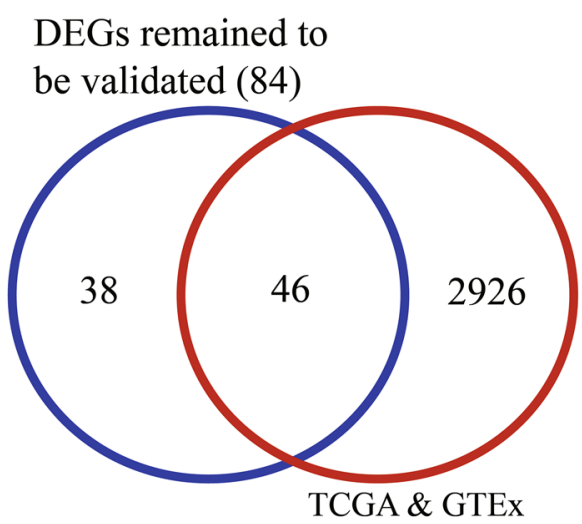

B

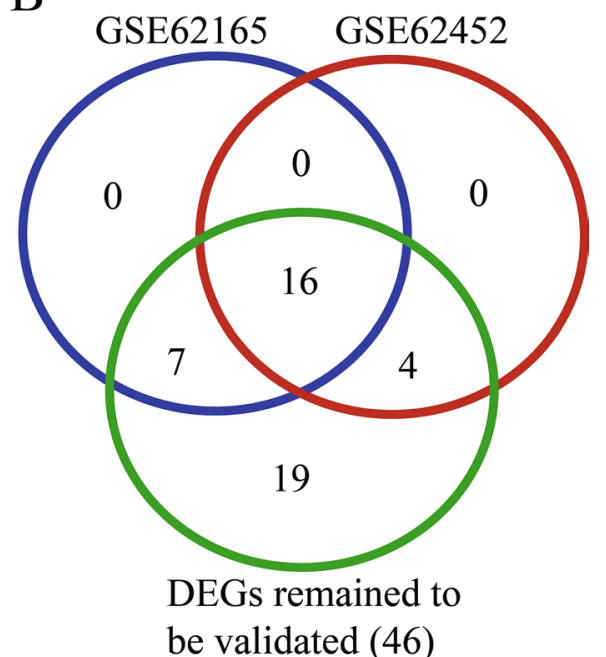

Fig. 5 Validation of DEGs between PDA and normal pancreatic tissue among TCGA, GTEx, and GEO datasets. A The intersection between the 84 DEGs in the GEO datasets and DEGs from the TCGA and GTEx datasets. Forty-six DEGs were identified after comparison of all three databases. B Validation of these 46 DEGs with GSE62165 and GSE62452. Sixteen out of 46 DEGs were confirmed. DEG differentially expressed gene, PDA pancreatic ductal adenocarcinoma, TCGA The Cancer Genome Atlas, GTEx Genotype-Tissue Expression, GEO Gene Expression Omnibus 


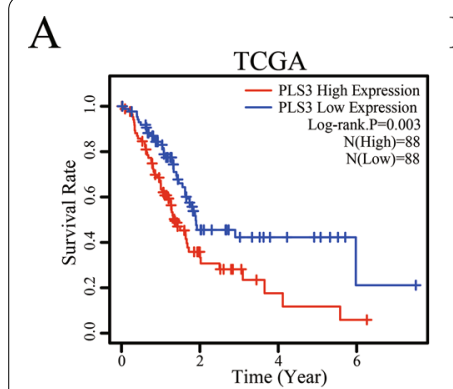

$\mathrm{E}$

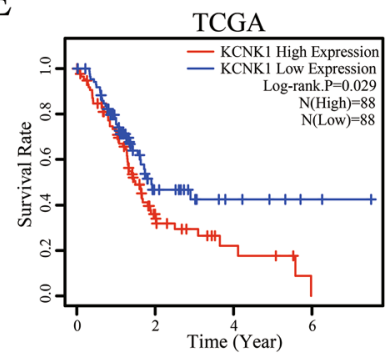

B

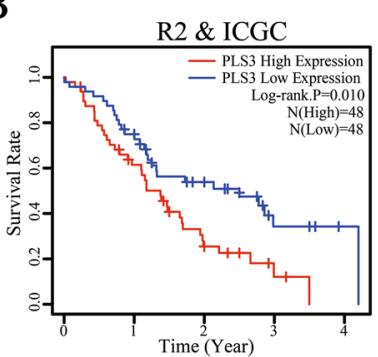

$\mathrm{F}$

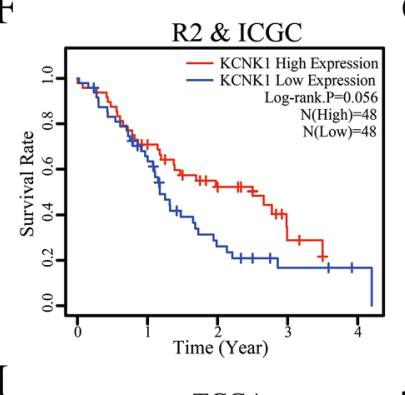

C

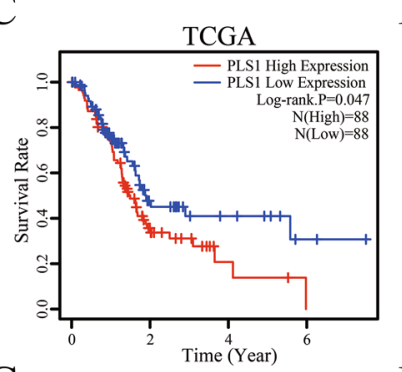

$\mathrm{G}$

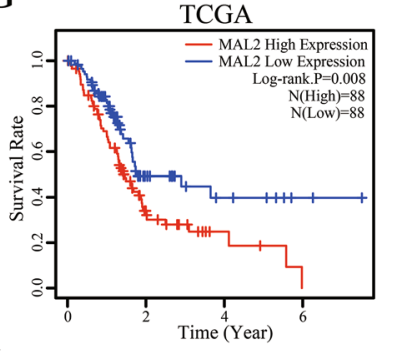

$\mathrm{D}$

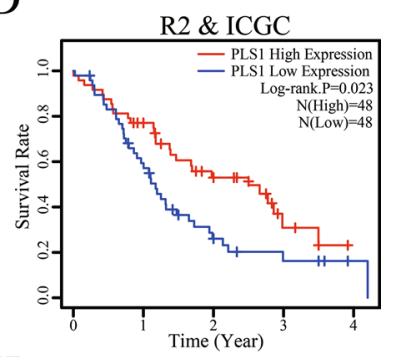

$\mathrm{H}$

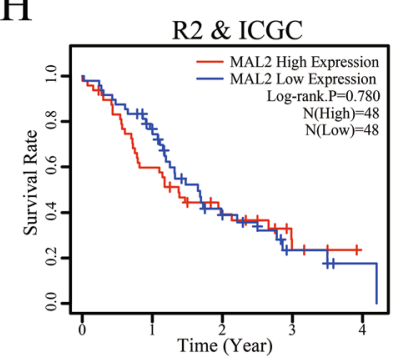

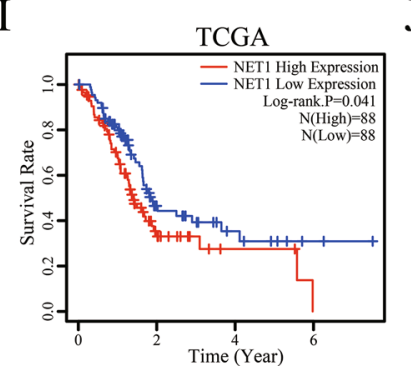

$\mathrm{J}$

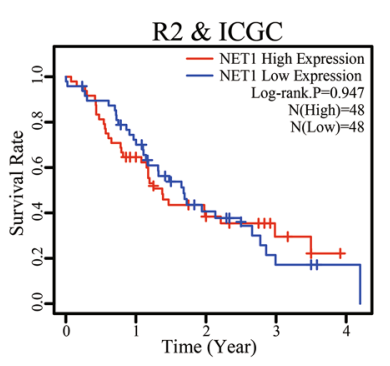

Fig. 6 Association of NET1, KCNK1, MAL2, PLS1, and PLS3 expression with PDA prognosis, as determined with TCGA, R2, and ICGC data. A, C, E, G, and I Kaplan-Meier curves and log-rank test results for PDA patients from TCGA. B, D, F, H, and $\mathbf{J}$ Kaplan-Meier curves and log-rank test results for PDA patients from the R2 and ICGC. Both studies show that patients with high PLS3-expressing PDA had poorer overall survival. PDA pancreatic ductal adenocarcinoma, TCGA The Cancer Genome Atlas, R2 The R2 Genomics Analysis and Visualization Platform, ICGC International Cancer Genome Consortium

while SPARC (secreted protein acidic and rich in cysteine) had a strong association with PLS3 expression in PDA (Fig. 9B-D). These patterns were also confirmed in TCGA PDA data (Fig. 9F-H). TCGA data revealed a weak association between KRT19 (another PDA marker, also known as CK19) with PLS3, while the GEO dataset showed a moderate association (Fig. 9E, I). We also performed Kaplan-Meier analysis. KRT7 was confirmed to be associated with prognosis in PDA patients in both datasets (Fig. 9J, N). KRT19 and SPP1 were validated by only one dataset (Fig. 9K, L, O, P). There was insufficient evidence to confirm the association between SPARC expression and OS in PDA patients (Fig. 9M, Q).

\section{Validation of differential PLS3 expression in different tissue} specimens

We performed RT-qPCR analysis of PLS3 in ten PDA samples, ten normal pancreas samples, five DLBCL samples, and five lymph node samples (Additional file 1:
Table S1). We found that the level of PLS3 mRNA in PDA was significantly higher than that in normal pancreas, DLBCL, and lymph node samples. PLS3 expression in normal pancreas was higher than that in DLBCL and lymph node samples. Intriguingly, PLS3 expression was similar in DLBCL and lymph nodes (Fig. 10).

\section{Discussion}

In the current study, we bioinformatically analyzed the DEGs among PDA, normal pancreas, DLBCL, and normal lymph node tissue samples by downloading the corresponding data from multiple online databases. We narrowed our analysis for association with PDA prognosis to those DEGs that occurred in all datasets, then focused on PLS3 as a biomarker for the early diagnosis of PDA, the prediction of prognosis, and differentiation from DLBCL. Our current study identified a great number of DEGs among these tissue samples, and our intersectional analysis narrowed them down to 84 DEGs, 
Table 5 Association of PLS3 expression with clinicopathological features in PDA patients from TCGA

\begin{tabular}{|c|c|c|c|c|}
\hline \multirow[t]{2}{*}{ Variables } & \multirow[t]{2}{*}{ Cases (number, \%) } & \multicolumn{2}{|c|}{ PLS3 level (number, \%) } & \multirow[t]{2}{*}{$P$} \\
\hline & & $\begin{array}{l}\text { High expression } \\
\mathrm{N}=83\end{array}$ & $\begin{array}{l}\text { Low expression } \\
\mathrm{N}=84\end{array}$ & \\
\hline \multicolumn{5}{|l|}{ Age (years) } \\
\hline$\leq 55$ & $33(19.8)$ & $20(60.6)$ & $13(39.4 \%)$ & \multirow[t]{2}{*}{0.16} \\
\hline$>55$ & $134(80.2)$ & $63(47.0)$ & $71(53.0)$ & \\
\hline \multicolumn{5}{|c|}{ Histologic grade } \\
\hline G1/G2 & $117(70.1)$ & $57(48.7)$ & $60(51.3)$ & \multirow[t]{2}{*}{0.69} \\
\hline G3/G4 & $50(29.9)$ & $26(52.0)$ & $24(48.0)$ & \\
\hline \multicolumn{5}{|c|}{ N classification } \\
\hline NO & $47(28.1)$ & $20(42.6)$ & $27(57.4)$ & \multirow[t]{2}{*}{0.24} \\
\hline N1 & $120(71.9)$ & $63(52.5)$ & $57(47.5)$ & \\
\hline \multicolumn{5}{|c|}{ T classification } \\
\hline $\mathrm{T} 1 / \mathrm{T} 2$ & $28(16.8)$ & $12(42.9)$ & $16(57.1)$ & \multirow[t]{2}{*}{0.42} \\
\hline $\mathrm{T} 3 / \mathrm{T} 4$ & $139(83.2)$ & $71(51.1)$ & $68(48.9)$ & \\
\hline \multicolumn{5}{|l|}{ Gender } \\
\hline Female & $76(45.5)$ & $37(48.7)$ & $39(51.3)$ & \multirow[t]{2}{*}{0.81} \\
\hline Male & $91(54.5)$ & $46(50.5)$ & $45(49.5)$ & \\
\hline \multicolumn{5}{|l|}{ Stage } \\
\hline Stage I/II & $160(95.8)$ & $79(49.4)$ & $81(50.6)$ & \multirow[t]{2}{*}{0.68} \\
\hline Stage III/IV & $7(4.2)$ & $4(57.1)$ & $3(42.9)$ & \\
\hline
\end{tabular}

Table 6 Univariate and multivariate Cox regression analyses of clinicopathological features associated with survival in PDA patients from TCGA

\begin{tabular}{|c|c|c|c|c|c|c|}
\hline \multirow[t]{2}{*}{ Parameter } & \multicolumn{3}{|c|}{ Univariate analysis } & \multicolumn{3}{|c|}{ Multivariate analysis } \\
\hline & HR & $\mathrm{HR} 95 \% \mathrm{Cl}$ & $\mathbf{P}$ & HR & $\mathrm{HR} 95 \% \mathrm{Cl}$ & $\mathbf{P}$ \\
\hline Age & 1.403 & $0.814-2.416$ & 0.121 & & & \\
\hline Histologic grade & 1.424 & $0.919-2.205$ & 0.113 & & & \\
\hline N classification & 2.180 & $1.283-3.706$ & 0.004 & 1.864 & $1.042-3.332$ & 0.036 \\
\hline T classification & 1.838 & $0.948-3.562$ & 0.071 & & & \\
\hline Gender & 0.781 & $0.514-1.187$ & 0.247 & & & \\
\hline Stage & 0.802 & $0.253-2.546$ & 0.708 & & & \\
\hline PLS3 expression & 1.566 & $1.028-2.385$ & 0.037 & 1.635 & $1.059-2.524$ & 0.026 \\
\hline
\end{tabular}

$H R$ hazard ratio, $\mathrm{Cl}$ confidence interval

Italicized value indicates $\mathrm{P}<0.05$

expression of which was significantly higher in PDA than in the three other types of tissue samples. These 84 upregulated DEGs were related to six different GO terms (the cell-cell junction, cell adhesion molecule binding, apical part of the cell, lateral plasma membrane, and desmosome). Moreover, cross-analysis of the TCGA data on 178 PDA and 171 normal pancreas samples verified 46 upregulated DEGs, 16 of which were significantly overexpressed in PDA; however, only five of these 16 DGEs showed significant association with poor PDA prognosis, and only PLS3 was further confirmed on R2 and ICGC database analysis. Our multivariate analysis confirmed that PDA N classification and PLS3 expression were independently predictors for OS among patients with PDA. PLS3 may also be used to diagnose PDA. In conclusion, our current data demonstrate that the detection of PLS3 expression may be used effectively to diagnose PDA, to predict OS in PDA patients, and to distinguish PDA from DLBCL. However, future study is warranted to verify our current data on the use of PLS3 as a biomarker for PDA.

PDA is one of the most malignant and lethal malignancies, and early diagnosis is crucial in controlling this deadly cancer. Surgical resection is the only method proven to control PDA clinically. Nevertheless, DLBCL 

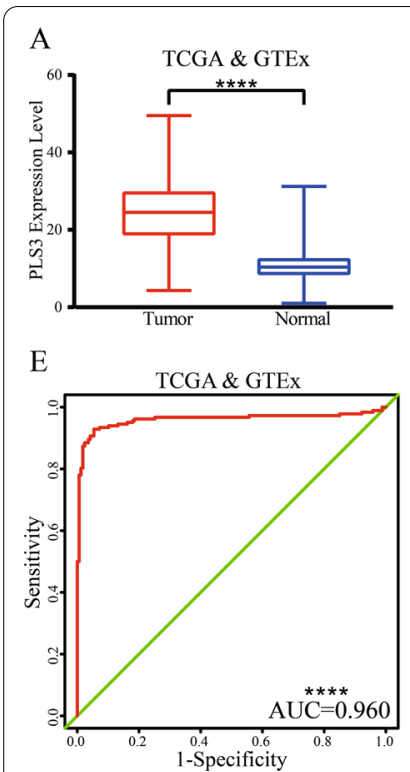
in all four datasets. E-H ROC curves for PLS3 expression in the four datasets. PDA pancreatic ductal adenocarcinoma, $R O C$ receiver operating characteristic curve. ${ }^{* * * *} \mathrm{P}<0.0001$ by Student's $t$-test

is usually treated with chemotherapy in the clinic. It is very important to make a differential diagnosis between PDA and pancreatic DLBCL because they have similar clinical appearance and medical imaging [11, 12, 20]. Due to the anatomic location of the pancreas, it is difficult to access and has a risk of intraoperative injury, while biopsy via surgery or laparoscopic surgery might not be the best choice. The EUS-FNA (endoscopic ultrasoundguided fine-needle aspiration biopsy) could be used to take a biopsy of peripancreatic masses, but as an invasive manipulation, the patients still have to take a risk of postoperative complications. Besides, a technical obstacle is an unavoidable issue [30]. By contrast, despite lower accuracy, serum tumor marker is non-invasive and easier to perform. To date, there have been numerous studies investigating biomarkers for PDA, including single markers [31] multiple biomarker panels [32], and immunebased proteomic panels [33]. For example, a recent study of oral flora showed that specific phylotypes were associated with increased risk for developing PDA [34]. Other recent work identified new-onset diabetes as a biomarker for early pancreatic cancer [35, 36].

$K$-Ras is mutated or altered in $95 \%$ of all PDA cases; p16 is mutated or altered in 95\%; p53 is mutated or altered in $75 \%$; Smad4 is mutated or altered in 55\% [37]. Mutations of any of these genes are associated with a poor PDA prognosis [3]. Regarding PLS3, one recent study that included 207 PDA tissue specimens showed that the overexpression of PLS3 was associated with tumor stage
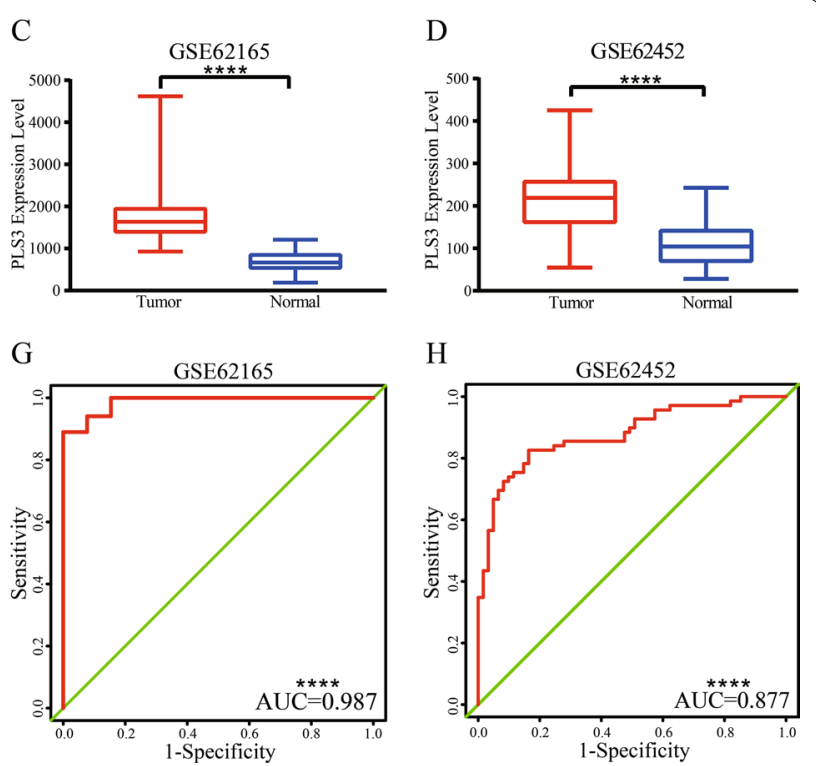

er than those of normal pancreas F

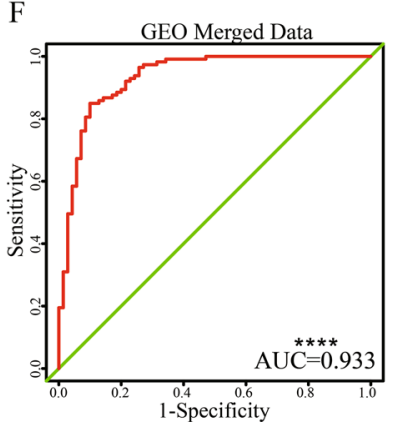

$P D A$ pancreatic ductal adenocarcinoma, $R O C$ receiver operating

and pathology as well as poor OS in PDA patients. PLS3 was an independent prognostic factor in PDA patients [14]. The authors' in vitro data demonstrated that PLS3 expression induced PDA cell proliferation and invasion, which were associated with increased PI3K/AKT activity in PDA cells [14]. Our current data support these recent reports of the role of PLS3 in PDA [14]. Nevertheless, there have been a number of studies of PLS3 in other human cancers, including gastric, colorectal, and breast cancers, as well as cutaneous T-cell lymphoma and acute myeloid leukemia [13-19].

PPL is an extremely rare form of malignant lymphoma with histology characteristic of DLBCL. Similarly to stage-matched DLBCL in the other organs, the condition can be treated with CHOP chemotherapy, which achieves equivalent outcomes [8]. One previous case report described the challenges in diagnosing DLBCL with an intra-sinusoidal pseudoglandular growth that mimicked poorly differentiated metastatic PDA in an intra-abdominal lymph node [38]. It is difficult to differentiate these DLBCLs from PDA [39, 40]. However, our current study did not include a direct comparison of the microarray data for PDA and the other three tissue types. Instead, we performed indirect analyses of differences in gene expression profiles between PDA and each one of the three other types. We believe that such analyses could be informative and the conclusion was validated by qPCR (Fig. 10). Our current study provided data on the differential diagnosis for PDA 


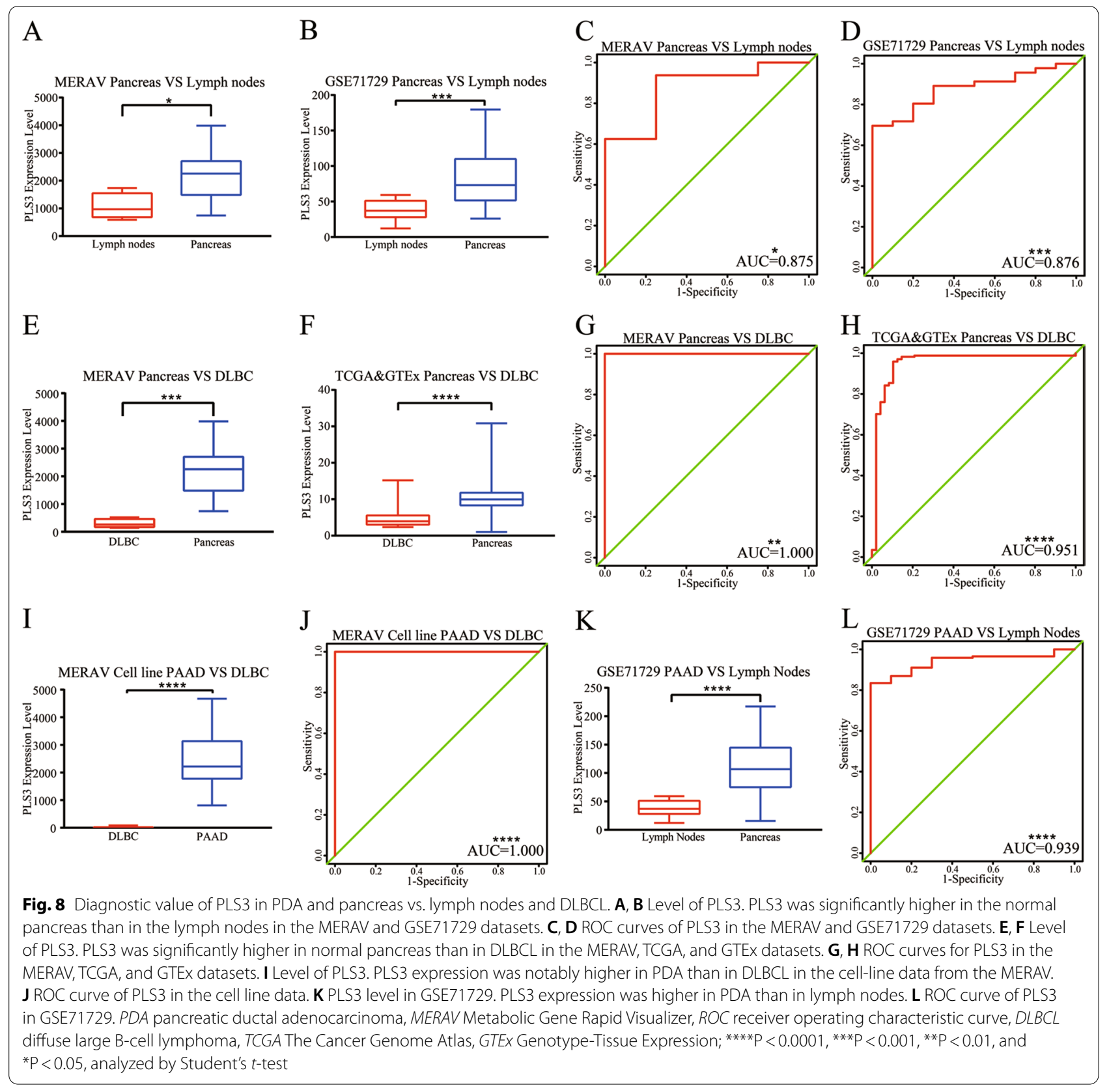

and DLBCL. We identified DEGs that were expressed at significantly higher levels in pancreas, compared with lymph nodes and DLBCL, including PLS3. PLS3 expression was also confirmed to significantly differ between PDA and the pancreas, which was consistent with a recent study of PLS3 in PDA [14]. Moreover, we applied bioinformatic methods to highlight the potential role of PLS3 as an improved PDA marker with

(See figure on next page.)

Fig. 9 Correlation of PLS3 with other PDA markers. A Correlation heatmap reporting Spearman correlation values for each comparison. The bar on the left represents the color legend of Spearman correlation values calculated for each paired PDA marker and PLS3 value. B-E The correlation of PLS3 with KRT7, KRT19, SPP1, and SPARC in the batched GEO dataset. F, IThe correlation of PLS3 with KRT7, KRT19, SPP1, and SPARC in TCGA. J-M The Kaplan-Meier curves and the log-rank test results of KRT7, KRT19, SPP1 and SPARC for PDA patients from TCGA. N-Q Kaplan-Meier curves and log-rank test results of KRT7, KRT19, SPP1 and SPARC for PDA patients from the R2 and ICGC. PDA pancreatic ductal adenocarcinoma, GEO Gene Expression Omnibus, TCGA The Cancer Genome Atlas, R2 The R2 Genomics Analysis and Visualization Platform, ICGC International Cancer Genome Consortium. ${ }^{* * *} \mathrm{P}<0.0001$ and ${ }^{* *} \mathrm{P}<0.001$ by Student's t-test 


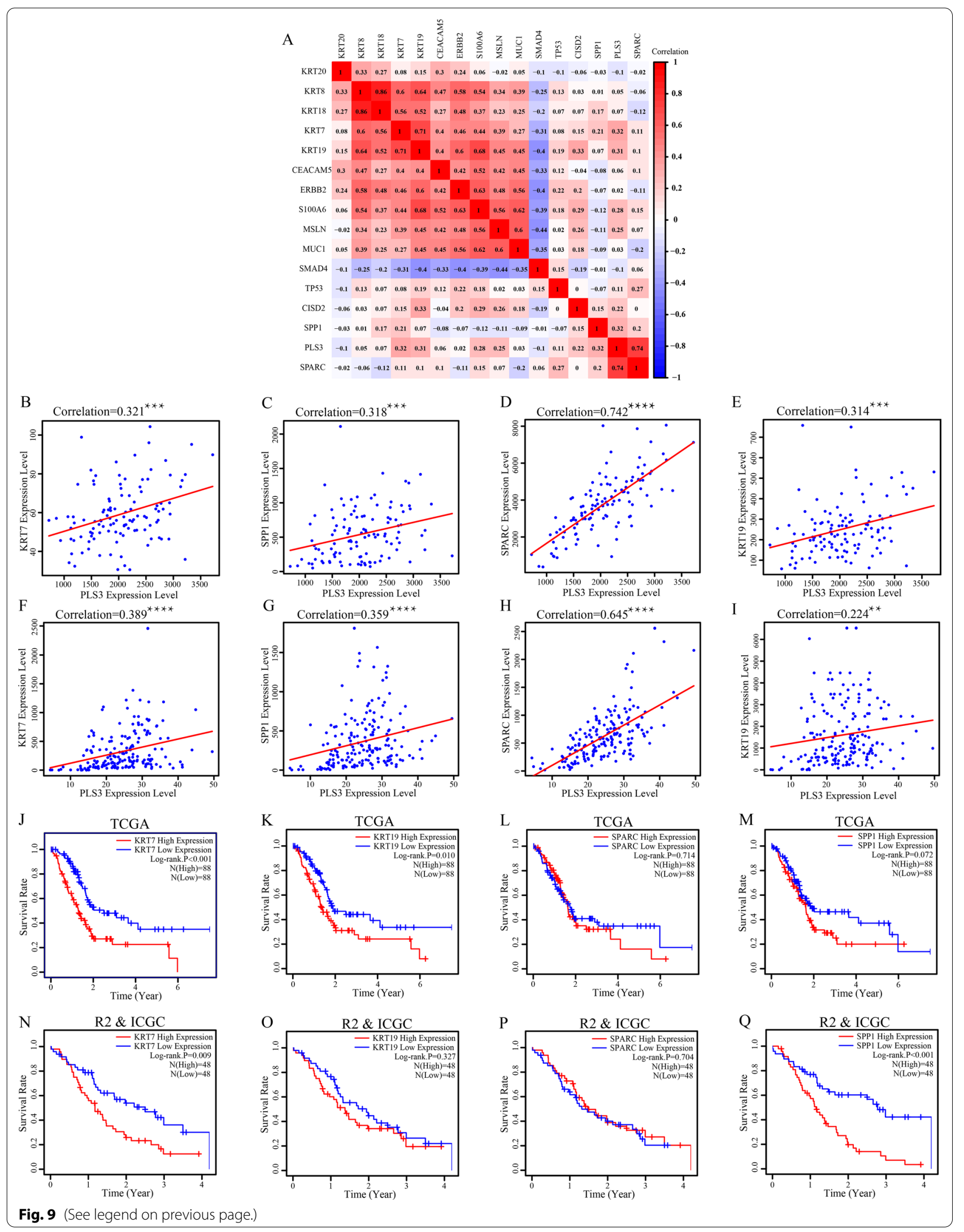




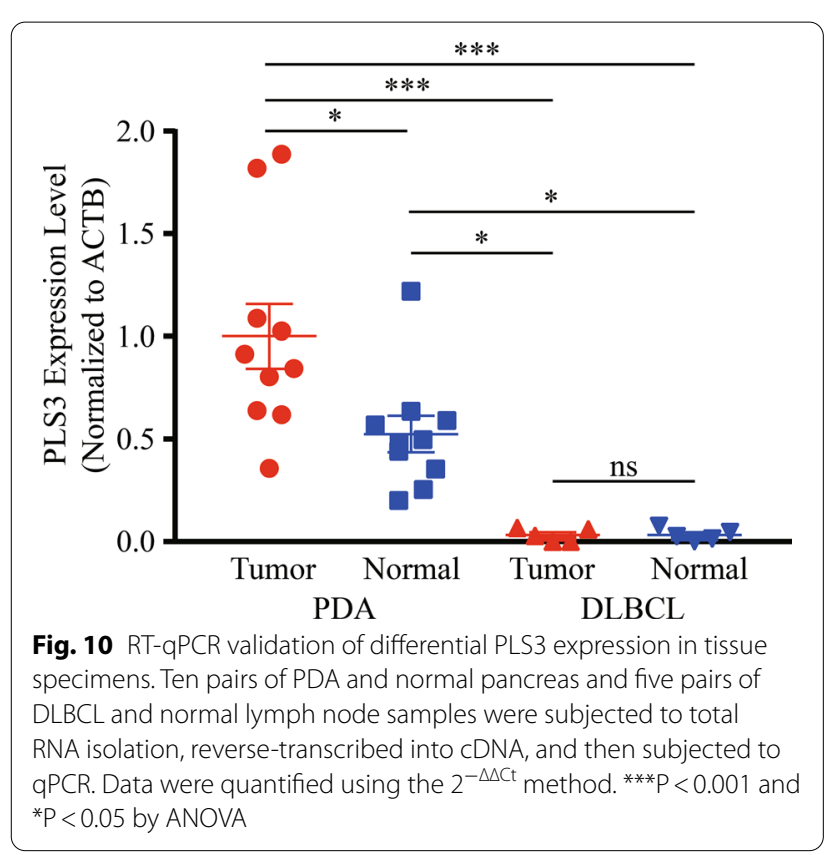

differentially diagnostic value for DLBCL that a traditional tumor marker may not have.

We further verified the association of PLS3 expression with other PDA biomarkers, like KRT7 and KRT19 [41], and SPARC and SPP1. For example, SPARC was reported to affect tumor cell proliferation and migration by activating PI3K/AKT signaling and the epithelial-mesenchymal transition in liver, lung, and head and neck cancers [42-44]. SPP1 was associated with the development of gastric cancer [45]. However, their interaction in PDA needs to be further investigated.

Overall, our current study has several advantages. We used the best of our knowledge to assess PLS3 as a biomarker to aid in the differential diagnosis of PDA from DLBCL. We endeavored to collect gene expression data from multiple databases, ultimately obtaining a total of 681 PDA, 361 normal pancreas, 69 DLBCL, and 14 lymph node samples, leading to a large sample size. Measurements of PLS3 are feasible because PLS3 levels can be detected in circulating tumor cells, indicating the potential value of PLS3 as a serum tumor marker [17, 46, 47]. However, our current study does have some limitations. For example, our current data did not allow for conclusions as to whether PLS3 overexpression occurred consistently in all PDA cells throughout our clinically heterogeneous population. Moreover, low levels of PLS3 expression may prevent accurate PPL diagnosis because of the inability to rule out the possibility of secondary pancreatic lymphoma. In addition, our current study did not include other types of pancreatic cancers, like neuroendocrine tumors, follicular lymphoma, and Hodgkin's lymphoma. Again, technically, our filter criteria for DEGs were extremely stringent, resulting in the inevitable loss of some useful information. Our current study utilized data from different online databases and datasets, leading to different numbers of cases and comparison groups, which sometimes made comparisons difficult. For example, we were only able to associate 16 DEGs (not 46 or 84) with patient survival (because these 16 DEGs were verified in the MERAV, GEO, and TCGA databases).

\section{Conclusions}

Our current study demonstrated that the detection of PLS3 expression may be useful in diagnosing PDA, predicting PDA prognosis, and distinguishing PDA from DLBCL. Further study will be needed to verify our current data on PLS3 as a biomarker or even as a novel target for PDA therapy.

\section{Abbreviations}

AUC: Area under the curve; DEG: Differentially expressed gene; DLBCL: Diffuse large B-cell lymphoma; EMT: Epithelial-mesenchymal transition; EUS-FNA: Endoscopic ultrasound-guided fine-needle aspiration biopsy; GEO: Gene Expression Omnibus; GO: Gene Ontology; GTEx:The Genotype-Tissue Expression; ICGC: International Cancer Genome Consortium; KCNK1: Potassium channel, two pore domain subfamily K, member 1; KRT19: Keratin 19; KRT7: Keratin 7; MAL2: Mal, T-cell differentiation protein 2; MERAV: Metabolic gEne RApid Visualizer; NET1: Neuroepithelial cell transforming 1; OS: Overall survival; PDA: Pancreatic ductal adenocarcinoma; PLS1: Plastin-1; PLS3: Plastin-3; PPL: Primary pancreatic lymphoma; ROC: Receiver operating characteristic curve; $r_{s}$ : Spearman's rank correlation coefficient; SPARC: Secreted protein acidic and cysteine rich; SPP1: Secreted phosphoprotein 1; TCGA: The Cancer Genome Atlas.

\section{Supplementary Information}

The online version contains supplementary material available at https://doi. org/10.1186/s12935-021-02117-1.

Additional file 1: Table S1. Clinicopathological data of the patients involved in Cox regression analysis, Kaplan-Meier analysis and RT-qPCR analysis. The data of TCGA, R2 and ICGC was downloaded from the web.

Additional file 2: Figure S1. Association of tumor N classification with PDA prognosis using TCGA database. TCGA data were downloaded from the web and statistically analyzed using the Kaplan-Meier curves and the log-rank test.

\section{Acknowledgements}

Not applicable.

\section{Authors' contributions}

YJC conceived and designed the experiments; FX prepared the manuscript; FX and GHW and BW analyzed the data. All authors read and approved the final manuscript.

\section{Funding}

This study was supported in part by grants from the National Natural Science Foundation of China (\#81572783 and \#81974438). The authors declare that they have no financial relationship with the organization that sponsored the research, and the funding body was not involved in study design, data collection, analysis and writing of the study. 


\section{Data availability}

The datasets generated and analyzed during the present study are available from the corresponding author on reasonable request.

\section{Declarations}

\section{Ethics approval and consent to participate}

The experimental protocol was established according to the ethical guidelines of the Helsinki Declaration and approved by the Ethics Committee of Tongji Hospital. All data published here is under the consent for publication.

\section{Consent for publication}

Not applicable.

\section{Competing interests}

The authors declare that there is no conflict of interest in this work.

Received: 6 May 2021 Accepted: 26 July 2021

Published online: 04 August 2021

\section{References}

1. Wild C, Weiderpass E, Stewart BW. Pancreatic cancer, world cancer report: cancer research for cancer prevention. Lyon: World Health Organization; 2020. p. 367-73.

2. Rahib L, Smith BD, Aizenberg R, Rosenzweig AB, Fleshman JM, Matrisian LM. Projecting cancer incidence and deaths to 2030: the unexpected burden of thyroid, liver, and pancreas cancers in the United States. Cancer Res. 2014;74:2913-21.

3. Wolfgang CL, Herman JM, Laheru DA, Klein AP, Erdek MA, Fishman EK, et al. Recent progress in pancreatic cancer. CA Cancer J Clin. 2013;63:318-48.

4. Vincent A, Herman J, Schulick R, Hruban RH, Goggins M. Pancreatic cancer. Lancet. 2011;378:607-20.

5. Kamisawa T, Wood LD, Itoi T, Takaori K. Pancreatic cancer. Lancet. 2016;388:73-85.

6. Urrutia G, Salmonson A, Toro-Zapata J, de Assuncao TM, Mathison A, Dusetti N, et al. Combined targeting of g9a and checkpoint kinase 1 synergistically inhibits pancreatic cancer cell growth by replication fork collapse. Mol Cancer Res. 2020;18:448-62.

7. Mukhija D, Nagpal SJS, Sohal DPS. Epidemiology, tumor characteristics, and survival in patients with primary pancreatic lymphoma: a large population-based study using the seer database. Am J Clin Oncol. 2019;42:454-8.

8. Zheng SM, Zhou DJ, Chen YH, Jiang R, Wang YX, Zhang Y, et al. Pancreatic T/histiocyte-rich large B-cell lymphoma: a case report and review of literature. World J Gastroenterol. 2017;23:4467-72.

9. Sadot E, Yahalom J, Do RK, Teruya-Feldstein J, Allen PJ, Gonen M, et al. Clinical features and outcome of primary pancreatic lymphoma. Ann Surg Oncol. 2015;22:1176-84.

10. Khashab M, Mokadem M, DeWitt J, Emerson R, Sherman S, LeBlanc J, et al. Endoscopic ultrasound-guided fine-needle aspiration with or without flow cytometry for the diagnosis of primary pancreatic lymphoma-a case series. Endoscopy. 2010;42:228-31.

11. Savari O, Al-Duwal Z, Wang Z, Ganesan S, Danan-Rayes R, Ayub S. Pancreatic lymphoma: a cytologic diagnosis challenge. Diagn Cytopathol. 2020;48:350-5.

12. Huang Z, Li M, He D, Wei Y, Yu H, Wang Y, et al. Two-dimensional texture analysis based on CT images to differentiate pancreatic lymphoma and pancreatic adenocarcinoma: a preliminary study. Acad Radiol. 2019;26:e189-95.

13. Kurashige J, Yokobori T, Mima K, Sawada G, Takahashi Y, Ueo H, et al. Plastin3 is associated with epithelial-mesenchymal transition and poor prognosis in gastric cancer. Oncol Lett. 2019;17:2393-9.

14. Xin Z, Li D, Mao F, Du Y, Wang X, Xu P, et al. PLS3 predicts poor prognosis in pancreatic cancer and promotes cancer cell proliferation via PI3K/AKT signaling. J Cell Physiol. 2020;235(11):8416-23.
15. Kujawski R, Przybyłowska-Sygut K, Mik M, Lewandowski M, Trzciński R, Berut $M$, et al. Expression of the pls3 gene in circulating cells in patients with colorectal cancer. Pol Przegl Chir. 2015;87:59-64.

16. Velthaus A, Cornils K, Hennigs JK, Grub S, Stamm H, Wicklein D, et al. The actin binding protein plastin-3 is involved in the pathogenesis of acute myeloid leukemia. Cancers. 2019;11:1663.

17. Ueo H, Sugimachi K, Gorges TM, Bartkowiak K, Yokobori T, Muller V, et al. Circulating tumour cell-derived plastin3 is a novel marker for predicting long-term prognosis in patients with breast cancer. $\mathrm{Br} J$ Cancer. 2015;112:1519-26.

18. Yokobori T, linuma $H$, Shimamura T, Imoto S, Sugimachi K, Ishii H, et al. Plastin3 is a novel marker for circulating tumor cells undergoing the epithelial-mesenchymal transition and is associated with colorectal cancer prognosis. Cancer Res. 2013;73:2059-69.

19. Szkandera J, Winder T, Stotz M, Weissmueller M, Langsenlehner T, Pichler $\mathrm{M}$, et al. A common gene variant in PLS3 predicts colon cancer recurrence in women. Tumour Biol. 2013;34:2183-8.

20. Shaul YD, Yuan B, Thiru P, Nutter-Upham A, McCallum S, Lanzkron C, et al. MERAV: a tool for comparing gene expression across human tissues and cell types. Nucleic Acids Res. 2016;44:D560-6.

21. Ritchie ME, Phipson B, Wu D, Hu Y, Law CW, Shi W, et al. limma powers differential expression analyses for RNA-sequencing and microarray studies. Nucleic Acids Res. 2015;43:e47.

22. Leek JT, Johnson WE, Parker HS, Jaffe AE, Storey JD. The sva package for removing batch effects and other unwanted variation in high-throughput experiments. Bioinformatics. 2012;28:882-3.

23. Yu G, Wang LG, Han Y, He QY. clusterProfiler: an R package for comparing biological themes among gene clusters. OMICS. 2012;16:284-7.

24. Walter W, Sanchez-Cabo F, Ricote M. GOplot: an R package for visually combining expression data with functional analysis. Bioinformatics. 2015;31:2912-4.

25. Bailey P, Chang DK, Nones K, Johns AL, Patch AM, Gingras MC, et al. Genomic analyses identify molecular subtypes of pancreatic cancer. Nature. 2016;531:47-52.

26. Robin X, Turck N, Hainard A, Tiberti N, Lisacek F, Sanchez JC, et al. pROC: an open-source package for R and S+ to analyze and compare ROC curves. BMC Bioinform. 2011;12:77.

27. Hornick JL, Lauwers GY, Odze RD. Immunohistochemistry can help distinguish metastatic pancreatic adenocarcinomas from bile duct adenomas and hamartomas of the liver. Am J Surg Pathol. 2005;29:381-9.

28. Huo Z, Zhai S, Weng Y, Qian H, Tang X, Shi Y, et al. PRPF40A as a potential diagnostic and prognostic marker is upregulated in pancreatic cancer tissues and cell lines: an integrated bioinformatics data analysis. Onco Targets Ther. 2019;12:5037-51.

29. Ma YY, Shi JJ, Chen JB, Xu KC, Niu LZ. Irreversible electroporation for liver metastasis from pancreatic cancer: a case report. World J Clin Cases. 2020;8:390-7.

30. Fritscher-Ravens A. Endoscopic ultrasound evaluation in the diagnosis and staging of lung cancer. Lung Cancer. 2003;41:259-67.

31. Kim J, Bamlet WR, Oberg AL, Chaffee KG, Donahue G, Cao XJ, et al. Detection of early pancreatic ductal adenocarcinoma with thrombospondin-2 and CA19-9 blood markers. Sci Transl Med. 2017. https://doi. org/10.1126/scitranslmed.aah5583.

32. Cohen JD, Javed AA, Thoburn C, Wong F, Tie J, Gibbs P, et al. Combined circulating tumor DNA and protein biomarker-based liquid biopsy for the earlier detection of pancreatic cancers. Proc Natl Acad Sci USA. 2017;114:10202-7.

33. Mellby LD, Nyberg AP, Johansen JS, Wingren C, Nordestgaard BG, Bojesen SE, et al. Serum biomarker signature-based liquid biopsy for diagnosis of early-stage pancreatic cancer. J Clin Oncol. 2018;36:2887-94.

34. Fan X, Alekseyenko AV, Wu J, Peters BA, Jacobs EJ, Gapstur SM, et al. Human oral microbiome and prospective risk for pancreatic cancer: a population-based nested case-control study. Gut. 2018;67:120-7.

35. Sharma A, Smyrk TC, Levy MJ, Topazian MA, Chari ST. Fasting blood glucose levels provide estimate of duration and progression of pancreatic cancer before diagnosis. Gastroenterology. 2018;155:490-500.e2. 
36. Sharma A, Kandlakunta H, Nagpal SJS, Feng Z, Hoos W, Petersen GM, et al. Model to determine risk of pancreatic cancer in patients with new-onset diabetes. Gastroenterology. 2018;155:730-739.e3.

37. Ryan DP, Hong TS, Bardeesy N. Pancreatic adenocarcinoma. New Engl J Med. 2014;371:1039-49.

38. Yau D, Aron M, Siddiqi IN. Diffuse large B-cell lymphoma with striking intrasinusoidal pseudoglandular growth pattern as a diagnostic dilemma mimicking metastatic poorly differentiated pancreatic adenocarcinoma in an intraabdominal lymph node. Int J Surg Pathol. 2019;27:181-4.

39. Constantin A, Tănase AD, Săftoiu A, Copăescu C. A primary retroperitoneal diffuse large b-cell lymphoma: a challenging diagnosis. Curr Health Sci J. 2018;44:392-6.

40. Ravindhran B, Prakash C, Govindharaj S, Bahnou NMS, Pavithra B. An aggressive primary retroperitoneal diffuse large B-cell lymphoma mimicking a pancreatic neoplasm, presenting as duodenal stenosis. J Clin Diagn Res. 2017;11:pd09-pd11.

41. Neureiter D, Zopf S, Dimmler A, Stintzing S, Hahn EG, Kirchner T, et al. Different capabilities of morphological pattern formation and its association with the expression of differentiation markers in a xenograft model of human pancreatic cancer cell lines. Pancreatology. 2005;5:387-97.

42. Chang CH, Yen MC, Liao SH, Hsu YL, Lai CS, Chang KP, et al. Secreted protein acidic and rich in cysteine (SPARC) enhances cell proliferation, migration, and epithelial mesenchymal transition, and SPARC expression is associated with tumor grade in head and neck cancer. Int J Mol Sci. 2017;18:1556
43. Wan S, Meyer AS, Weiler SME, Rupp C, Toth M, Sticht C, et al. Cytoplasmic localization of the cell polarity factor scribble supports liver tumor formation and tumor cell invasiveness. Hepatology. 2018;67:1842-56.

44. Sun W, Feng J, Yi Q, Xu X, Chen Y, Tang L. SPARC acts as a mediator of TGFbeta1 in promoting epithelial-to-mesenchymal transition in A549 and H1299 lung cancer cells. BioFactors. 2018;44:453-64.

45. Song SZ, Lin S, Liu JN, Zhang MB, Du YT, Zhang DD, et al. Targeting of SPP1 by microRNA-340 inhibits gastric cancer cell epithelial-mesenchymal transition through inhibition of the PI3K/AKT signaling pathway. J Cell Physiol. 2019;234:18587-601.

46. Shi F, Ma Y, Qian Y, Wang Y, Wang Z, Zhao M, et al. A novel peptide probe for identification of PLS3-expressed cancer cells. Anal Chem. 2019;91:9640-7.

47. Markiewicz A, Topa J, Nagel A, Skokowski J, Seroczynska B, Stokowy T, et al. Spectrum of epithelial-mesenchymal transition phenotypes in circulating tumour cells from early breast cancer patients. Cancers. 2019;11:59.

\section{Publisher's Note}

Springer Nature remains neutral with regard to jurisdictional claims in published maps and institutional affiliations.
Ready to submit your research? Choose BMC and benefit from:

- fast, convenient online submission

- thorough peer review by experienced researchers in your field

- rapid publication on acceptance

- support for research data, including large and complex data types

- gold Open Access which fosters wider collaboration and increased citations

- maximum visibility for your research: over 100M website views per year

At BMC, research is always in progress.

Learn more biomedcentral.com/submissions 